\title{
Psychotherapy as Heuristic Search
}

\author{
Joachim Diederich \\ Psychology Network Pty Ltd \\ Level 1, 16 McDougall St \\ Milton Q1d 4064 Australia \\ joachim@psychologynetwork.com.au \\ School of Information Technology and Electrical Engineering \\ The University of Queensland \\ Brisbane Qld 4072 Australia
}

\begin{abstract}
There is no coherent framework for psychological therapy available. From psychoanalysis to behaviour therapy, humanistic and third wave approaches, there are significant differences in terms of aetiology, methods and expected outcomes. Heuristic search, an important method in artificial intelligence (AI), can serve as a general frame of reference for psychotherapy. This approach is based on a clear definition of the starting and end points of psychological therapy and how to achieve identified outcomes; it allows the utilisation of particularly effective psychological interventions and a ranking of different forms of psychotherapy with regard to efficacy, time and effort. A cornerstone of this framework is the notion of "deep heuristic", that is, particularly effective psychological interventions based on clinical experience. It is likely that the heuristic search framework can be applied to interventions in psychiatry as well as health disciplines in general.
\end{abstract}

\section{Introduction}

Heuristic search is a form of problem solving. There is a clearly identified start state (e.g. a client with a set of symptoms) and a goal state (e.g. the client is free of symptoms). Often there are multiple or complex goals (e.g. client is free of symptoms and well supported by a social network). There are also means of transforming one state to another: psychological interventions. These interventions are informed by evidence-based research but clinical practice is important as well. A significant part of the training of clinical psychologists is based on clinical practice. An experienced clinician develops heuristic knowledge: facts, skills and methods that are particularly useful. Sometimes this knowledge is explicit, for instance, when the therapists knows that $\mathrm{s} /$ he can initiate a certain action; sometimes the knowledge is implicit, that is, there is no conscious awareness of it. Here, deep heuristics refer to psychological interventions that are deemed particularly useful based on clinical experience in a given context.

Psychological interventions are the means of transforming one state (set of symptoms, level of well being) to another in order to achieve a clearly defined goal. Psychological interventions can be ranked at any point in time with regard to a number of criteria: (1) Legal requirements and the management of risks, (2) empirical support for the intervention, (3) probability of success and (4) 
confidence of the practitioner to conduct the intervention based on training and clinical experience. Clearly, the ranking can include other criteria as well.

In summary, artificial intelligence developed the idea that problem solving is search. From this viewpoint, psychotherapy is the search for means to achieve goal states; freedom of symptoms frequently among them. The solution to a problem is a viable path from a starting point (the client presents with symptoms) to one or several goals states (absence of symptoms, general well being, social support etc).

\section{Motivation}

The objective here is to offer a unifying approach to psychological therapy. The framework introduced here facilitates a comparison of different forms of psychotherapy that do not share common ground with regard to aetiology and the nature of psychological interventions. Furthermore, the framework considers and integrates knowledge obtained from empirical studies as well as from clinical practice. A new approach for the measurement of efficacy is introduced that acknowledges the diversity of psychological interventions and yet focuses on observable and measurable outcomes. A component of this framework is the preregistration of psychological treatment similar to empirical, psychological research. The clinician is asked to nominate a set of assessment methods, psychological interventions and intended outcomes and the result of psychological treatment is then evaluated in the context of that registration.

Traditionally, problem-solving therapy includes adaptive problem-solving skills to cope with a stressful problem (American Psychological Association, Division 12, 2018). However, it is possible to go much further and see problem solving as a general framework for psychological therapy.

While psychological assessment, and in particular psychometric testing, has a firm theoretical, mathematical basis, no such coherent and unifying framework is available for psychological therapy. Heuristic search can serve as a general framework for psychotherapy. A heuristic is a "rule of thumb", a method that might not always find the best solution but is guaranteed to find a good solution in reasonable time. Heuristic search is a form of problem solving that aims at finding the best and most cost-effective path from a starting point to a well-defined goal. This approach is based on a clear definition of the starting and end points of psychological therapy and how to achieve identified outcomes; it allows the utilisation of particularly effective psychological interventions and a ranking of different forms of psychotherapy with regard to time and effort.

Daily clinical practice includes the observation of the behaviour of a client, partners, care-givers and families. It includes the understanding and appropriate response to facial expressions, gestures, the use of metaphors and sometimes slang language. Much of this knowledge is embodied in the sense that clients use their full presence to communicate and the psychologist is using speech, voice, gesture etc. to ask questions and to conduct psychological interventions.

As a result, the "knowledge base" of a clinical psychologist is not only a large network of facts but "a large array of informal judgement rules (heuristics) which guide the system (here: the clinical psychologist) toward plausible paths to follow and away from implausible ones (Lenat, 
1982, p.189). This path includes the individual steps of psychological assessment and therapy. Furthermore, this heuristic knowledge cannot be directly observed, however, it frequently is the foundation of daily, clinical practice.

For instance, an experienced psychologists may use "goal decomposition" as a strategy within the general context of problem-solving therapy. Based on clinical experience and an understanding of the problem, the psychologist may suggest sub-goals in order to achieve the overall outcome (the goal). For instance, if the psychologist has worked with clients who are on the autistic spectrum, the practitioner may may well have a set of heuristics to develop social communication that can help to achieve sub-goals as well as the final outcome.

\section{Case Studies}

In order to illustrate the utility of the framework, a number of case studies are summarised below and the process of psychotherapy is translated into the conceptual structure introduced here.

\section{$\underline{\text { 3.1.1 Historic Case Studies }}$}

\subsection{Little Albert}

Little Albert is among the most famous case studies in psychology. Watson \& Rayner (1920) used classical conditioning to demonstrate how a form of "neurosis" can develop in a child (Trull $\&$ Prinstein, 2013). A young boy who was not afraid of any furry animals was presented with a white rat and while he was playing with the animal a loud noise was made behind him. After few trials, Albert developed a fearful response and this generalised to other furry objects (the ethics of this study and the experimental set-up have been severely criticised for decades).

Jones (1924; in Trull \& Prinstein, 2013) demonstrated how fear can be removed in an independent study with a three-year old boy. Peter was afraid of rabbits and similar animals. In order to treat this form of anxiety, Jones (1924) brought a caged rabbit closer and closer to the boy as he was eating. As a result, the dreaded object became associated with food and after some period of time, Peter's fear of rabbits disappeared. It was important that Jones (1924) assessed the fear of rabbits to make sure it was not so intense that the child would develop an aversion to food. This tightly controlled treatment was successful.

Jones (1924) had a clearly defined starting point for her psychological therapy: Peter and his fear of rabbits. In addition, there are methods which are now part of behaviour therapy to eradicate the fear (in this framework, these are the means to move from one sub-goal to the next). The starting point was Peter with a set of symptoms (fear of a rabbit). The goal state was the client free of any symptoms. Step-by-step, Jones (1924) brought the cage with the rabbit closer and closer to Peter. By doing so, she moved from the start state to intermediate states and finally to the goal state (a client relieved of this particular fear). Please note that while the individual actions (moving the cage) are almost identical, the intermediate problem solving states in this particular form of therapy differ. Each state has a different probability to develop an aversion to food! Assessment was required to make sure that this food aversion did not happen. Clearly, an intermediate state with a higher probability of an aversion to food is less desirable than a state that brings the client closer to a freedom of symptoms with low probability of developing 
negative side effects. Peter's treatment can be clearly defined with the problem solving framework.

\subsubsection{Little Hans}

This very famous case study by Sigmund Freud is about childhood anxiety as well. Hans' (not his real name) father belonged to Freud's circle of followers in Vienna and he noted that Hans developed a fear of horses. The father wrote to Freud: "No doubt the ground was prepared by sexual over-excitation due to his mother's tenderness; but I am not able to specify the actual exciting cause. He is afraid a horse will bite him in the street, and this fear seems somehow to be connected with his having been frightened by a large penis" (Strachey, 1909). Alternative explanations for the fear of horses have been offered, including the observation that five-year old Hans may have been frightened by a collapsing horse on the street.

Freud had a sexual interpretation of the fear of horses broadly based on the concept of the Oedipus complex. With this understanding Freud started the process of psychoanalysis. There was no follow-up, however, fourteen years later Hans reported to be fine and well-adjusted without memory of his childhood phobia.

Freud stated that psychoanalysis is a therapeutic measure and not a scientific investigation (Wolpe \& Rachman, 1960, p.145). It appears that that psychoanalysis, and in particular the interpretations, provided the means to achieve goals such as freedom of symptoms. In this case, no fear of horses.

\subsection{A fictional, contemporary case study: Maryam}

Maryam was referred by her mother Hibba as she is exhibiting anger and conduct problems at home. Hibba perceived Maryam to be a challenging child since birth. At the age of 2, parenting advice was sought from a psychologist since Hibba was overwhelmed by the experience of having a child and this resulted in arguments with her partner Omar as well. Both parents found it difficult to respond positively to Maryam and aversive interaction patterns emerged, between both parents we well as the parent-child system. The aversive behaviour of her parents provided modelling opportunities for Maryam and her self-esteem deteriorated in recent years since her academic performance is below average. There are significant protective factors: Maryam lives in a stable home environment; she is physically healthy, both parents are employed and have sought help for Maryam in the past. In addition, no conduct problems at school have been reported. Maryam's behaviour should improve if the family commits to a psychological treatment based on cognitive behaviour therapy (CBT) and improvements in the family system.

The following long-term goals have been identified:

1. To reduce Maryam's anxiety, in particular separation anxiety, as well as feelings of panic due to separation fears.

2. To reduce periods of low and irritable mood as well as episodes of anger. 
The long-term goals will be achieved if there is (1) a significant reduction in anxiety and depression scores (self- and parent assessment; T-scores below 60), and (2) a reduction in observer-rated scores for mood problems. This goal should be achieved after 4-6 months (8-12 sessions).

In addition, the following mid-term goals have been identified: To increased periods away from mother Hibba without expressed fear or anger as well as sleeping in Maryam's own bedroom on the other side of the house. The mid-term goal will be achieved if (1) Maryam attends day-time play-dates on her own twice a week and (2) sleeps in her own bedroom several times a week (after 4-8 weeks). Progress towards these goals will be monitored during the first 2-8 sessions (every session).

Finally, here are the short-term goals for therapy: Maryam to identify negative thinking patterns related to separation anxiety and replace these with more positive thoughts. This will be measured by Maryam expressing these skills during sessions and completing homework assignments for two consecutive sessions. Maryam should achieve these goals within 2-4 weeks (to be monitored every session).

After identifying clear goals for psychotherapy, the means for transitioning from one state to another are identified. In this case, CBT and family therapy. Barrett et al. (2001, p.135/6) observe that CBT alone as well as CBT plus family management/treatment showed greater improvement on a variety of measures at a 12-month follow-up when compared with a group without treatment. More specifically, "clients that received family training also showed significantly greater improvement than the CBT-only group on a number of measures" (Barrett et al., 2001, p.136).

Byng-Hall (1995, p. 6) argues that a family therapist can provide a "temporarily secure base for the whole family during therapy". The overall aim is to "use this secure base to help the family explore ways of improving the security of the family's attachment network" (Byng- Hall, 1995, p.7). The family can then use the secure base to establish closer and more satisfying relationships.

After the identification of goals and sets of psychological interventions that allow to move from one intermediate state to another, the sessions can proceed as follows:

a) First session (with Maryam and both parents): Learning to beat anxiety. The foundations of CBT are explained to Maryam and Hibba.

b) Second session (with Maryam): Introduction of the "Hot Diary" (a booklet with negative, problematic thoughts). Several sample situations are discussed.

c) Third session (with the entire family: Explain attachment theory and "circle of security". Also, explain the importance of a "secure family base".

d) Fourth session (with Maryam). Introduction to controlled breathing exercises. Discussion of the Hot Diary and fears in relation to sleeping in Maryam's own bed.

In this case study, goal decomposition is used to determine the desired final outcome but also to identify sub goals that constitute progress in psychotherapy. For benchmarking purposes, it is clearly stated which goals should have been reached after a certain number of sessions. Interventions from cognitive behaviour therapy as well as family therapy are the actions that 
allow to traverse problem space, that is to move from one sub-goal to the next until the final outcome is achieved. In addition, the criteria for achieving goal state are very clear: scores in psychological tests that measure child anxiety.

\section{Knowledge acquisition and automated psychotherapy: Kriton Speech}

Kriton Speech integrates cognitive computing and psychological methods to acquire knowledge for artificial intelligence systems and to apply this knowledge for computational diagnosis and therapy. Kriton was developed as a general knowledge acquisition system and has been adopted for psychological applications (Diederich 1987a,d, 1988). As a knowledge acquisition system, Kriton Speech elicits domain specific knowledge and as an application, it applies this knowledge for diagnosis and therapy. Kriton Speech exclusively uses a voice user interface (VUI). The system uses knowledge bases such as upper-level ontologies as well as domain-specific knowledge and psychological heuristics (1) to interview the user to obtain knowledge and based on this knowledge (2) perform tasks such as psychological assessment and therapy. The system allows free verbal responses and has an explanation capability, i.e. provides feedback about why certain questions are asked. Speech and language are analysed to determine, with high accuracy, if there is a mental health problem.

Attention economy is a concept in information management that treats human attention as a limited commodity. Herbert A. Simon (1971) is considered to be the first person having introduced the concept of attention in economics. Simon (1971) outlined that in an information rich world there is a limitation of the resource that consumes information: human attention. Almost half a century later, we are now at the point where there are so many information sources, so many devices and so many distractions that human attention is not only limited, it is frequently impaired.

Kriton Speech, an artificial intelligence system, addresses both problems: the limitation of human attention and the challenge of growing knowledge. Firstly, Kriton Speech uses speech only to interact with the user and therefore does not require any visual attention. This enables the user to direct attention to other information sources and to perform physical actions while using the program. No learning is required, any user can interact with Kriton Speech immediately. It is possible to drive a car while talking to Kriton Speech; to go for a walk or to perform other activities.

Kriton Speech is using upper-level ontologies as well as existing domain-specific knowledge to interview the user. The employment and use of already acquired knowledge has major advantages: (1) The interview aims at completing and refining existing knowledge in addition to the acquisition of new concepts and rules. (2) On the application side, the system utilises up-todate clinical knowledge obtained from practising mental health experts to conduct psychological assessment and therapy.

As a result of the interview, Kriton Speech builds and verifies ontologies and rule based systems. The output is a Web Ontology Language (OWL) file that can be edited by the use of standard ontology editors. Information obtained from clients is stored in an XML format to be used by eHealth systems. 


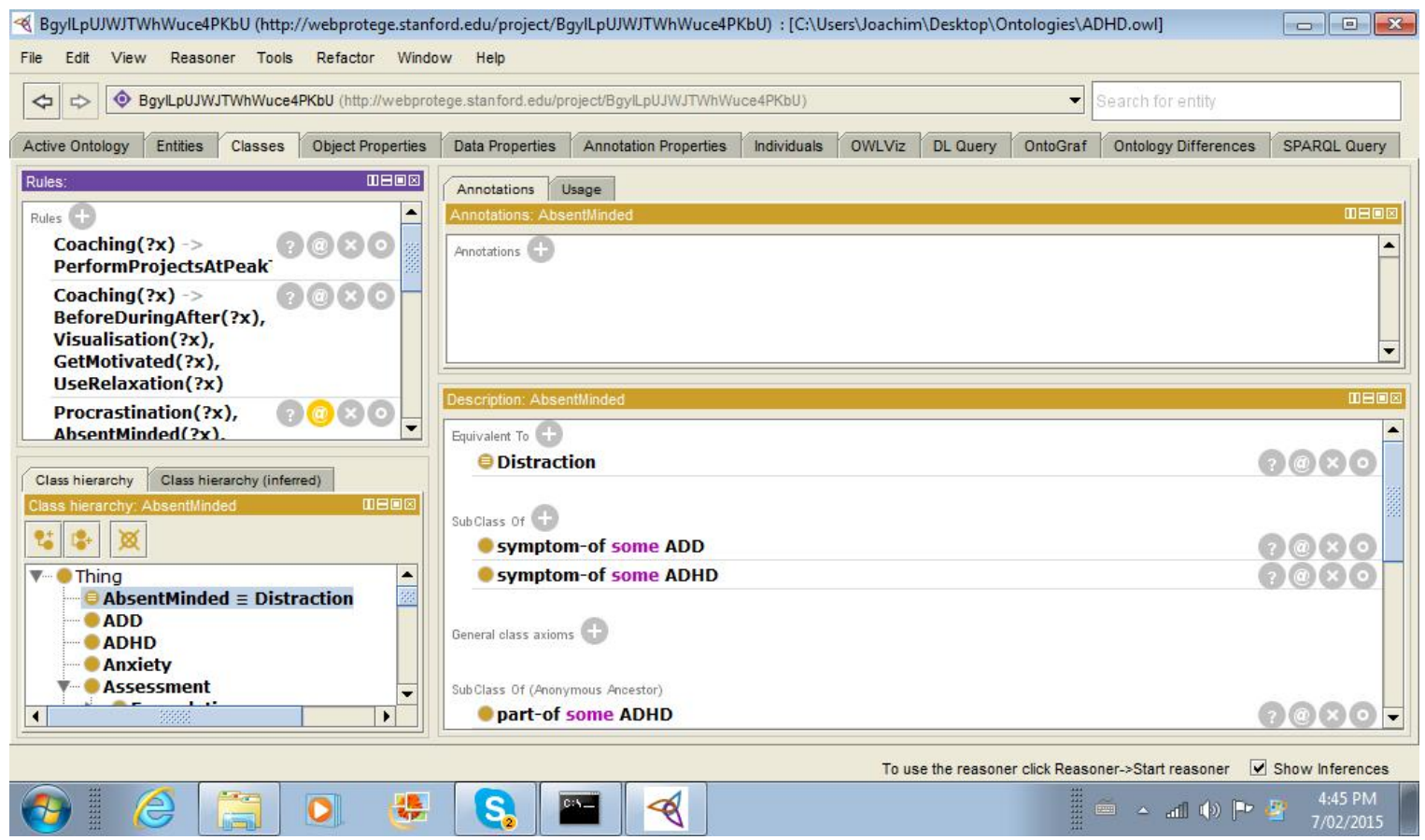

Figure 1: An ontology for ADHD coaching.

\subsection{Amy: An artificial intelligence ADHD coach}

Amy is an artificial intelligence (AI) system that provides psychological assistance simply by talking to a client. Amy is available 24 hours a day and provides an additional dimension for psychological service delivery. When linked to background information in the form of ontologies, Amy has extensive medical and psychological knowledge and the built-in capacity to acquire additional knowledge from mental health experts. In turn, this knowledge is used for psychoeducation. Amy's primary mode of communication is a simple and convenient conversation, however, Amy also provides videos, images and text to educate users about mental health issues.

Amy operates in a number of different modes. If the user is inclined to have a free conversation about any topic or to obtain information about ADHD, Amy is simply a chatbot with a very strong protection against inappropriate content. With machine learning enabled, Amy detects mood changes in speech and text including those pointing to depression and can modify the interaction accordingly. Longer conversations can be analysed to extract personality characteristics of the user. While Amy has the ability to learn, the dialogue structure is rulebased and does not change. As a result, Amy is always socially and culturally appropriate. Amy frequently refers to online resources to illustrate recommendations, including the use of relaxation techniques.

\subsubsection{Technical Solution.}

Many of the current "artificial psychologist" solutions go wide, i.e. they aim at providing counselling on almost any subject. As a result, these solutions do not have in-depth knowledge 
about mental health disorders and they cannot provide therapy that is specifically tailored to address complex psychological conditions. The approach here is narrow: Amy uses extensive medical and psychological knowledge bases, including knowledge provided by practising clinical psychologists. All these knowledge sources are used to perform tasks such as psychoeducation and coaching but only in the context of ADHD! Amy's knowledge bases are updated frequently to include the latest facts about mental health and ADHD. Currently, Amy is interacting in English language. Multi-lingual implementations in a number of Asian language are expected soon. Amy interacts primarily through social media (e.g. Twitter).

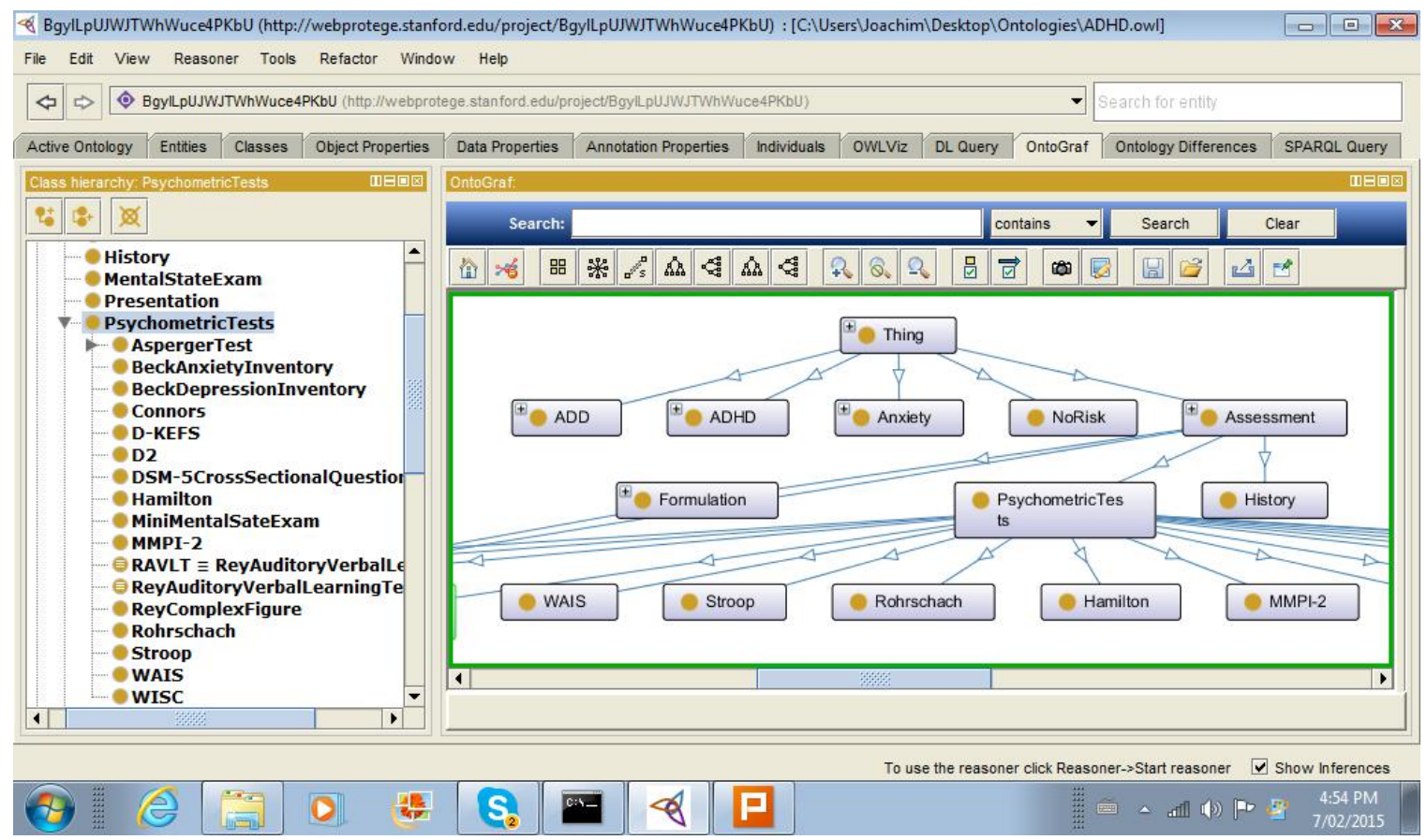

Figure 2: A generic ontology for clinical psychology.

\section{Conclusion}

The framework introduced here applies to all forms of psychological service deliver, from the clinical practise of trained psychologists to computational approaches. Heuristic search can serve as a general frame of reference for psychotherapy. This approach is based on a clear definition of the starting and end points of psychological therapy and how to achieve identified outcomes; its usefulness is demonstrated by use of case studies. It is likely that the heuristic search framework can be applied to interventions in psychiatry as well as health disciplines in general. The approach allows the utilisation of particularly effective psychological interventions and a ranking of different forms of psychotherapy with regard to time and effort.

\section{References}

Barrett, P. M., Duffy, A. L., Dadds, M. R., \& Rapee, R. M. (2001). Cognitive-behavioral treatment of anxiety disorders in children: long-term (6-year) follow-up. Journal of Consulting and Clinical Psychology, 69, 135-141. 
Diederich, J., Techniken des Wissenserwerbs (Knowledge Acquisition Techniques), in Christaller, Th. (Ed.), 5. Künstliche Intelligenz Frühjahrsschule (1987). Berlin: Springer Verlag $1988=$ Springer Informatik Fachberichte 202, 295-335.

Diederich, J., Ruhmann, I., May, M., KRITON: A Knowledge Acquisition Tool For Expert Systems. International Journal of Man-Machine Studies 26 (1987) 29-40.

Diederich, J., Ruhmann, I., May, M., KRITON: A Knowledge Acquisition Tool for Expert Systems, in Boose, J.H., Gaines, B. (Eds.), Knowledge Acquisition Tools for Expert Systems. Knowledge-Based Systems 2. London etc: Academic Press (1988) 83-94.

Lenat, D.B., The Nature of Heuristics. Artificial Intelligence, 19 (1982) 189-249.

Strachey, J. Analysis of a Phobia in a Five-Year-Old Boy. The Standard Edition of the Complete Psychological Works of Sigmund Freud, Volume X (1909): Two Case Histories ('Little Hans' and the 'Rat Man'), 1-150.

Sijbrandija, M., Acarturkb, C., Birdc, M., Bryantd, R.A., Burcherte, S. ...... Strengthening mental health care systems for Syrian refugees in Europe and the Middle East: integrating scalable psychological interventions in eight countries. European Journal of Psychotraumatology, 2017, VOL. 8, 1388102 https://doi.org/10.1080/20008198.2017.1388102.

Simon, H. A. (1971) "Designing Organizations for an Information-Rich World" in: Martin Greenberger, Computers, Communication, and the Public Interest, Baltimore. MD: The Johns Hopkins Press. pp. 40-41.

Trull, T.J., Prinstein, M.J. Clinical Psychology (Eighth Edition). Wadsworth CENGAGE Learning, Belmond CA, 2013.

What is Problem-Solving Therapy? American Psychological Association | Division 12 http://www.div12.org/sites/default/files/WhatIsProblemSolvingTherapy.pdf Retrieved 22 March 2018.

Wolpe, J., Rachman, S. Psychoanalytic "evidence": A critique based on Freud's case of little Hans. Journal of Nervous and Mental Disease, 131 (1960) 135-148. 